\title{
Modeling of the Saturnian Ring System
}

\author{
Evgeny Griv, Edward Liverts, Michael Gedalin \\ Department of Physics, Ben-Gurion University, Beer-Sheva, Israel \\ Chi Yuan \\ Institute of Astronomy, Academia Sinica, Taipei 106, Taiwan
}

\begin{abstract}
A self-consistent system of the Boltzmann equation and the Poisson equation is used to study the dynamical evolution of Saturn's main $\mathrm{A}, \mathrm{B}$, and $\mathrm{C}$ rings. The theory, as applied to the Saturnian ring system, predicts for several features, such as numerous irregular density wakes, with size and spacing between them of the order $4 \pi \rho \approx 2 \pi h$, where $\rho$ is the mean epicycle radius of the particle and $h$ is the typical thickness of the system under study. In Saturn's rings, $\rho \lesssim 10 \mathrm{~m}$. Computer $N$-body experiments are desribed which test the validities of the theory. Use of the 112-processor SGI Origin 2000 supercomputer is enabled us to make long runs using a large number of particles in the direct simulation code and thus simulate phenomena not previously studied numerically. We predict that forthcoming in 2004 Cassini spacecraft high-resolution images will reveal this recurrent fine-scale $\sim 100 \mathrm{~m}$ or so structure in low and moderately high optical depth regions of the rings.
\end{abstract}

\section{Introduction}

Saturn's rings disk of mutually gravitating particles is highly dynamic and is subject to various instabilities of gravity disturbances. This is because the evolution of the system is primarily driven by angular momentum redistribution. This might take place through global mechanisms like nonaxisymmetric instabilities caused by self-gravity, which develop in the equatorial plane of the system. ${ }^{1}$ See Goldreich \& Tremaine (1982) as a review of the problem.

In the current research, the theory of small-amplitude collective oscillations (of the Lin-Shu type invoked to explain the spiral structure of disk galaxies; Lin \& Shu 1966, Lin et al. 1969, and Shu 1970) and their stability of Saturn's rings disk is developed. We use the method of gas-kinetic theory by employing the kinetic formalism of magnetized plasma theory which deals with similar problems. We test the validity of the theory by $N$-body calculations. The results of the analysis are applied to Saturn's rings, composed of rock and ice: we

\footnotetext{
${ }^{1}$ In addition, bending waves, external, and embedded Saturn's moons also play a role in sculpting its elaborate ring system (Goldreich \& Tremaine 1982).
} 
predict the recurrent structure of order of $100 \mathrm{~m}$ (or even less) to be observed in the low and moderately high optical depth regions of the rings A, B, and C. In fact, it should be made clear right from the start that the suggestion of fine-scale $\lesssim 100 \mathrm{~m}$ structure in Saturn's rings due to the effect of self-gravity is not an entirely new idea. Apparently, the microstructure in Saturn's rings has already been predicted by local simulations (Salo 1992; Osterbart \& Willerding 1995; Richardson 1994; Griv 1998; Daisaka \& Ida 1999). Earlier, the so-called quadrupole asymmetry observed in the A ring has been explained in terms of numerous unresolved spiral density "wakes" by Colombo et al. (1976) and Franklin et al. (1978). The presence of wakes causes the effective area covered by particles, hence brightness, of the ring to vary at different longitudes. Our significant contribution is just a kinetic theory derivation and a direct simulation of results obtained before in local simulations.

\section{Kinetic Theory of Small-Amplitude Gravity Perturbations}

In Saturn's rings, the local distribution function $f(\mathbf{r}, \mathbf{v}, t)$ of particles must satisfy the familiar Boltzmann kinetic equation (e.g., Griv et al. 2000):

$$
\frac{\partial f}{\partial t}+\mathbf{v} \cdot \frac{\partial f}{\partial \mathbf{r}}-\frac{\partial \Phi}{\partial \mathbf{r}} \cdot \frac{\partial f}{\partial \mathbf{v}}=\left(\frac{\partial f}{\partial t}\right)_{\mathrm{St}}
$$

In Eq. (1), $\Phi(\mathbf{r}, t)$ is the total gravitational potential determined self-consistently from the Poisson equation $\nabla^{2} \Phi=4 \pi G \int f d \mathbf{v}$. Also, $(\partial f / \partial t)_{\mathrm{St}}$ is the so-called collision integral which takes into account effects due to the discrete-point nature of the gravitational charges. We use the simple kinetic model when the exact, but complicated, Boltzmann integral $(\partial f / \partial t)_{\text {St }}$ is replaced by an approximate, phenomenological term in the form of the Krook model (Shu \& Stewart 1985; Griv \& Chiueh 1997; Griv et al. 2000): $(\partial f / \partial t)_{\text {St }}=-\nu_{\mathrm{c}}\left(f-f_{0}\right)$, where $f_{0}$ is the steady-state Maxwellian-like equilibrium distribution function and $\nu_{\mathrm{c}}$ plays the role of the velocity-independent collision frequency.

Boltzmann equation (1) and the Poisson equation give a complete description of the problem for disk modes of collective oscillations. We simplify this system of equations by considering the epicyclic, local WKB, and asymptotic Lin-Shu approximations (see Griv et al. 1999 and Griv et al. 2000 for details). As a result, the generalized dispersion relation may be obtained:

$$
\frac{k^{2} c_{r}^{2}}{2 \pi G \sigma_{0}|k|}=-\kappa \sum_{l=-\infty}^{\infty} l \frac{e^{-x} I_{l}(x)}{\omega_{*}-l \kappa+i \nu_{\mathrm{c}}}+2 \Omega \frac{m \rho^{2}}{r L} \sum_{l=-\infty}^{\infty} \frac{e^{-x} I_{l}(x)}{\omega_{*}-l \kappa+i \nu_{\mathrm{c}}}
$$

where $k=\sqrt{k_{r}^{2}+m^{2} / r^{2}}$ is the wavenumber, $c_{r}$ is the radial dispersion of random velocities, $\sigma_{0}$ is the equilibrium surface mass density, $\kappa$ is the epicyclic frequency, $I_{l}(x)$ is the modified Bessel function of the order $l, x=k_{*}^{2} c_{r}^{2} / \kappa^{2} \approx k_{*}^{2} \rho^{2}, k_{*}$ is the effective wavenumber, $\rho \approx c_{r} / \kappa$ is the epicyclic radius, $\omega_{*}=\omega-m \Omega$ is the Doppler-shifted (in a rotating frame) wavefrequency, $m$ is the number of spiral arms, $\Omega$ is the angular velocity (in Saturn's rings $\kappa \approx \Omega$ ), and $L$ is the radial scale of a spatial inhomogeneity. In Eq. (2), the second term on the right-hand side is the small correction, $\rho^{2} / r|L| \ll 1$. The dispersion relation (2) 

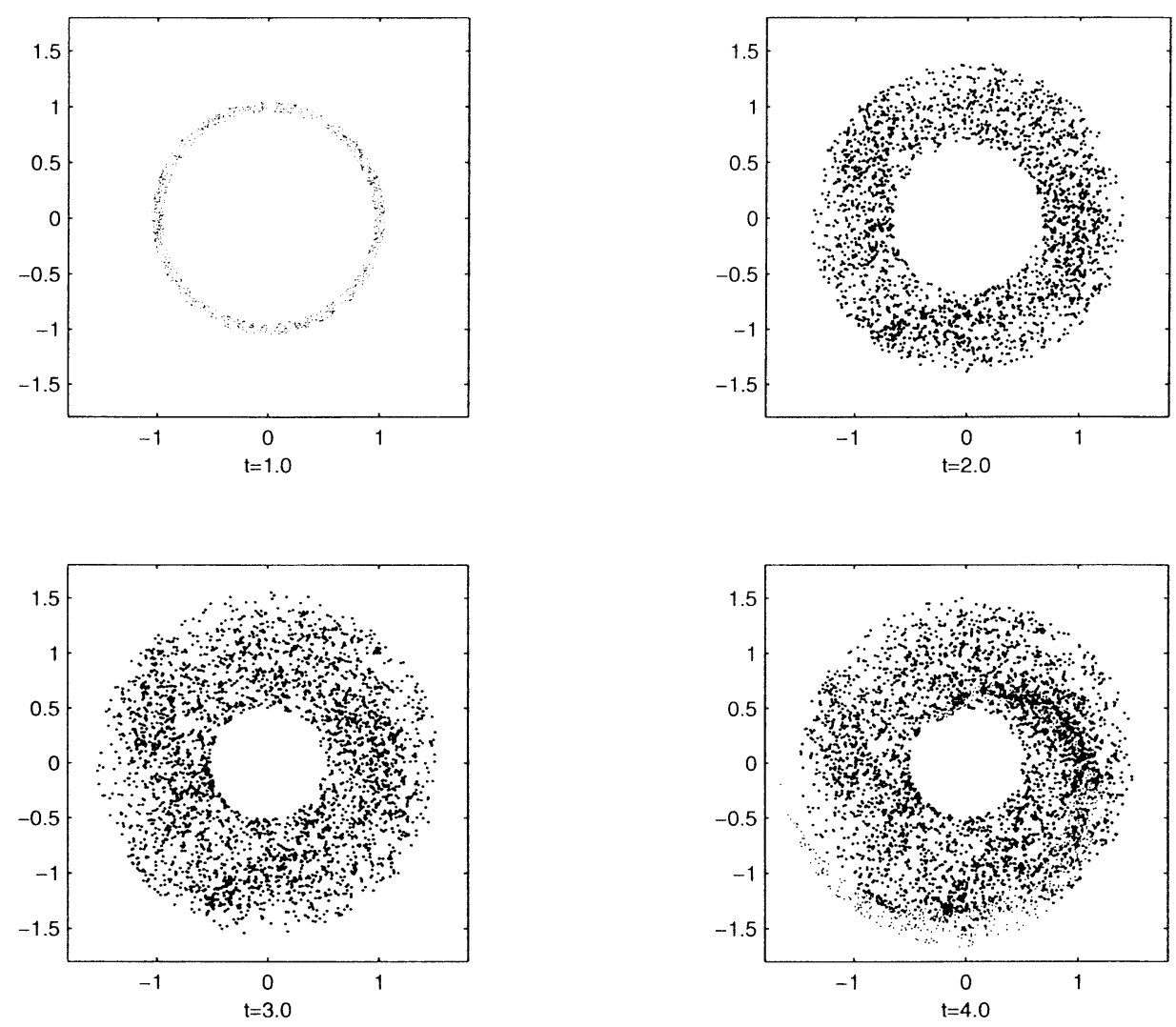

Figure 1. The time evolution of a narrow ring of identical particles with rare interparticle collisions. The direction of the disk rotation was taken to be counterclockwise, and time $t=1$ corresponds to a single revolution of the disk. The system is violently Jeans-unstable.

describes the ordered behavior of a medium near its metaequilibrium state and generalizes the ordinary Lin-Shu dispersion relation (Lin \& Shu 1966; Lin et al. 1969; Shu 1970) for nonaxisymmetric Jeans perturbations, $m \neq 0$, to the case where physical collisions occur in an inhomogeneous disk. ${ }^{2}$

The dispersion relation (2) is complicated: the basic dispersion relation above is highly nonlinear in the frequency $\omega_{*}$. To see the physical meaning of solutions of Eq. (2), one does not need the exact solutions. Rather, in order to deal with the most interesting oscillation types, let us consider various limiting

\footnotetext{
${ }^{2}$ Generally, the term "Jeans instabilities" identifies nonresonant instabilities associated with aperiodically growing accumulations of mass (cf. electrostatic bunching instabilities or a firehose instability in a plasma). In plasma physics an instability of the Jeans-type is known as the negative-mass instability of a relativistic charged particle ring or the diocotron instability of a nonrelativistic ring that caused rapid azimuthal clumping of beams in synchrotrons, betatrons, and mirror machines.
} 
cases of perturbations described by some simplified variations of Eq. (2). For instance, similar to the plasma physics method, it is sufficient to only consider the principal part of the disk between the inner and outer Lindblad resonances, $|l| \leq 1$ (Lin \& Shu 1966; Lin et al. 1969; Shu 1970; Griv et al. 2000). As a result, Eq. (2) can be represented in the simplest form

$$
\left(\omega_{*}+i \nu_{\mathrm{c}}\right)^{3}-\left(\omega_{*}+i \nu_{\mathrm{c}}\right) \omega_{J}^{2}+\omega_{\mathrm{gr}} \kappa^{2}=0
$$

where the square of the Jeans frequency is $\omega_{\mathrm{J}}^{2}=\kappa^{2}-2 \pi G \sigma_{0}|k| F(x), F(x) \propto$ $\exp (-x)$ is the so-called "reduction factor," which takes into account the fact that the wave field affects only weakly the particles with high random velocities, and $\omega_{\mathrm{gr}}=2 \Omega e^{-x} I_{0}(x)\left(2 \pi G \sigma_{0}|k| / k^{2} c_{r}^{2}\right)\left(m \rho^{2} / r L\right)$ is the frequency of the so-called gradient oscillations. Equation (3) describes two ordinary Jeans branches of oscillations - the most important long-wavelength branch, $x=k_{*}^{2} c_{r}^{2} / \kappa^{2} \approx k_{*}^{2} \rho^{2} \lesssim$ 1 , and the short-wavelength one, $x>1$ - and a gradient branch of oscillations modified by collisions (Griv et al. 2000).

From relation (3) in the frequency range $\left|\omega_{*}^{3}\right| \sim\left|\omega_{\mathrm{J}}^{3}\right| \gg\left|\omega_{\mathrm{gr}}\right| \kappa^{2}$ the dispersion law for the Jeans branch of oscillations is

$$
\omega_{* 1,2} \approx \pm p\left|\omega_{\mathrm{J}}\right|-\omega_{\mathrm{gr}} \frac{\kappa^{2}}{2 \omega_{\mathrm{J}}^{2}}-i \nu_{\mathrm{c}}
$$

where $p=1$ for Jeans-stable perturbations with $\omega_{\mathrm{J}}^{2}>0$ and $p=i$ for Jeansunstable perturbations with $\omega_{\mathrm{J}}^{2}<0$. In Eq. (4), the terms involving $\omega_{\mathrm{gr}}$ and $i \nu_{\mathrm{c}}$ are the small corrections, and in general $\left|\omega_{* 1,2}\right| \approx\left|\omega_{\mathrm{J}}\right| \sim \kappa$. From Eq. (4) one concludes that both Jeans-stable and Jeans-unstable perturbations will weakly decay as a result of rare collisions. Accordingly, a spatial inhomogeneity will not influence the stability condition of Jeans modes. It follows that the Jeansunstable perturbations grow almost aperiodically, $\left|\Re \omega_{* 1,2} / \Im \omega_{* 1,2}\right| \ll 1$. The gradient of macroscopic parameters and azimuthal mode number $m$ determine the small real part of such hydrodynamically (Jeans-) unstable modes.

The Jeans perturbations can be stabilized by the random velocity spread. Indeed, if one recalls that such unstable perturbations are possible only when $\omega_{* 1,2}^{2} \approx \omega_{\mathrm{J}}^{2}<0$, then by using the condition $\omega_{* 1,2}^{2} \geq 0$ for all possible $k$, a stability criterion against arbitrary Jeans perturbations can be written in the form (Griv et al. 2000)

$$
c_{r} \geq c_{\mathrm{T}}\left\{1+\left[(2 \Omega / \kappa)^{2}-1\right] \sin ^{2} \psi\right\}^{1 / 2} \approx c_{\mathrm{T}}\left[1+3 \sin ^{2} \psi\right]^{1 / 2}
$$

where $\tan \psi=m / k_{r} r$ and $c_{\mathrm{T}} \approx 3.4 G \sigma_{0} / \kappa$ is the well-known Toomre's (1964) critical velocity dispersion to suppress the instability of only axisymmetric (radial) gravity perturbations. The stability criterion thus obtained represents generalization of Toomre's (1964) criterion to the case when additionally nonaxisymmetric (spiral) gravity perturbations are taken into account. The parameter $\left\{1+\left[(2 \Omega / \kappa)^{2}-1\right] \sin ^{2} \psi\right\}^{1 / 2}$ is an additional stability parameter that depends on both the pitch angle $\psi$ and the amount of differential rotation in the disk $d \Omega / d r$ (cf. the parameter $\mathcal{J}$ introduced by Lin \& Lau 1979 and Bertin 1980). As one can see from Eq. (5), the modified critical velocity dispersion 
$c_{\text {crit }}$ grows with $\psi$. Consequently, in order to suppress the most "dangerous," in the sense of the loss of gravitational stability, very open nonaxisymmetric perturbations with $\psi>45^{\circ}, c_{\text {crit }}$ should obey the following modified stability criterion: $c_{r} \geq c_{\text {crit }}=(2 \Omega / \kappa) c_{\mathrm{T}} \approx 2 c_{\mathrm{T}}$. Collective motion connected with the Jeans-unstable mode is excited in the plane of a disk when the random velocity dispersion is not sufficiently large, $c_{r} \leq 2 c_{\mathrm{T}}$, in other words, if the effective Toomre's $Q$-value is $Q \leq 2 \Omega / \kappa \approx 2$.

It follows from Eq. (3), the wavelength of maximum Jeans instability $\lambda_{\text {crit }}=$ $2 \pi / k_{\text {crit }}$ is given by (Griv et al. 2000)

$$
\lambda_{\text {crit }} \approx(2 \Omega / \kappa) 2 \pi \rho \approx 4 \pi c_{r} / \kappa
$$

For the parameters of Saturn's rings $\lambda_{\text {crit }} \sim 100 \mathrm{~m}$, and such scales of a few hundred meters correspond to the predicted fine-scale structure of Saturn's rings.

\section{Direct $N$-body Simulations of Saturn's Rings}

The generalized dispersion relation (2), the stability criterion (5), and the critical wavelength (6) obtained in the framework of the linear kinetic theory do not reveal precisely what kind of structure can emerge due to the gravitational instability. Computer simulations should be able to identify these structures. Different methods may be employed to simulate the evolution of the particulate disk of the Saturnian ring system by $N$-body experiments. For instance, following Salo (1992) one can study some aspects of disk dynamics by applying the numerical approach of local $N$-body simulations. The most serious criticism of such model calculations is that too much of the relevant physics has been left out for such a relatively simple method (Griv et al. 1999). In this work, we develop an algorithm for a direct simulation code. The difficulty is that the speed of most individual computers is still too limited for the number of model particles in a direct simulation. That is, owing to the circumstance that the computing time increases as the square of the number of attracting points, there is definite practical upper limit to this number $N \sim 1000$. There is now a broad consensus that major discoveries in dynamics of planetary rings would be within grasp if computers at least 100 times faster than today's conventional workstations were available, assuming equal progress in algorithms and software. Thus, the most serious criticism of direct calculations is that a small number of particles might not permit revelation of the fine-scale structure (see Griv \& Chiueh 1998 for a discussion). The problem is solved by using the modern parallel supercomputers: with the multiple processors in supercomputers it is possible to reduce the running time by processing more than one group of particles at a time. The use of, say, 100 parallel computers would make such calculations practical. In the present study, we explore the 112-processor SGI Origin 2000 system of the Inter University Computational Center in Israel. We are able to use up to 200,000 particles in long-term direct runs.

We simulate the evolution of a model for Saturn's rings by direct integration of the equations of motion of particles. The force on a particle $i$ due to all other 

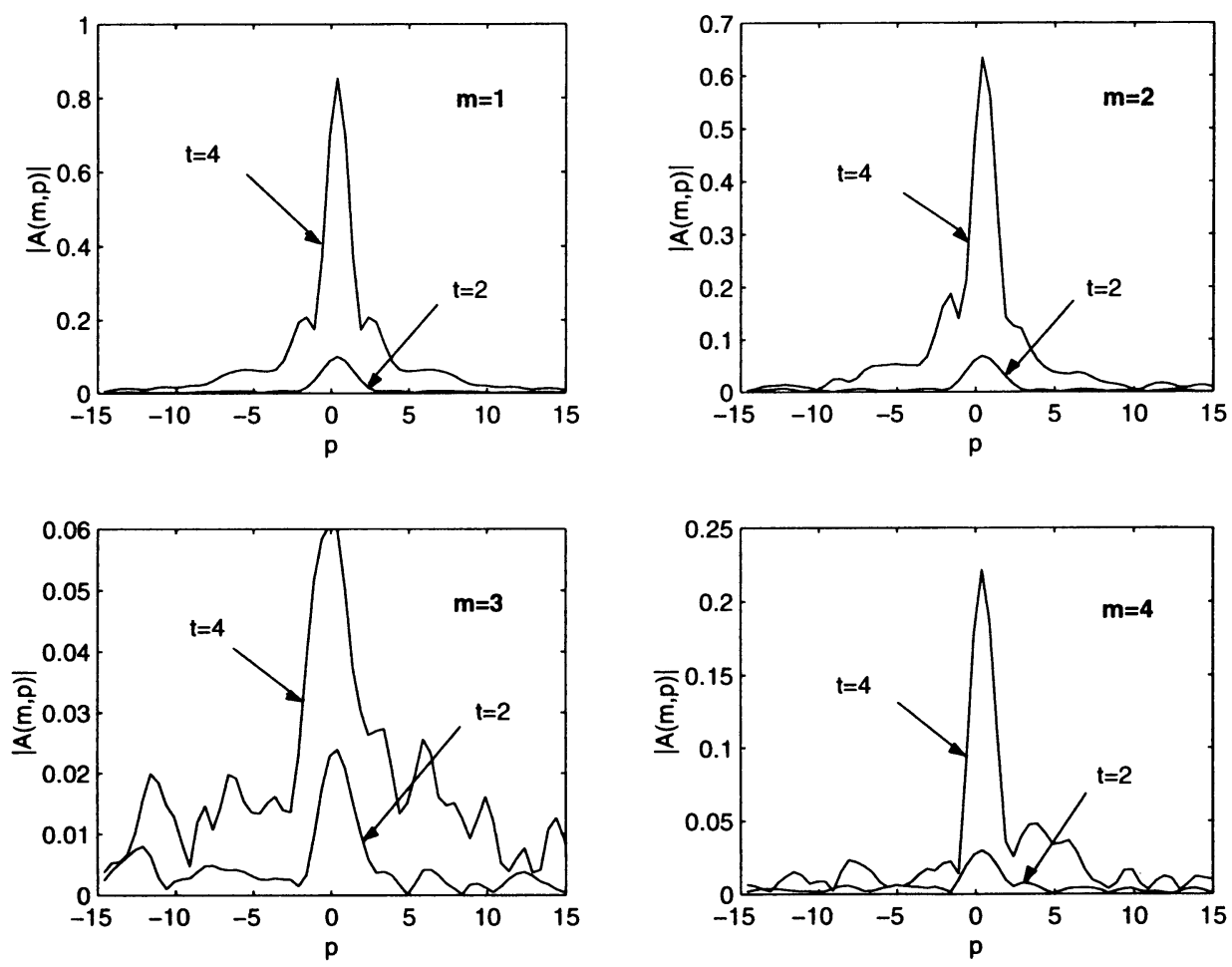

Figure 2. The time evolution of the Fourier spectrum of the particle distribution shown in Figure 1 for different azimuthal mode numbes $m$.

particles is given by

$$
\frac{d^{2} \mathbf{r}_{i}}{d t^{2}}=G m_{i} m_{j} \sum_{j \neq i}^{N} \frac{\left(\mathbf{r}_{j}-\mathbf{r}_{i}\right)}{\left[\left(\mathbf{r}_{j}-\mathbf{r}_{i}\right)^{2}\right]^{3 / 2}}
$$

where $G$ is the constant of gravitation, $m_{i}, m_{j}, \mathbf{r}_{i}$ and $\mathbf{r}_{j}$ are the mass, position and velocity of the $i$-th and of the $j$-th particle, respectively. The model with rare interparticle collisions is considered: $\nu_{\mathrm{c}} \ll \Omega$. Our integration algorithm is based on Griv \& Chiueh (1998) $N$-body integrator, which uses the standard RungeKutta method and individual time-step schema in the integration of particle orbits. Some modifications, taking advantage of the ring-shaped geometry and inelastic particle-particle impacts have been made.

The particles were initially placed on nearly circular orbits with an anisotropic Schwarzschild distribution of small radial, azimuthal, and vertical random velocities components: $c_{r}=2 c_{\varphi}, c_{z}=0.1 c_{r}$, and $c_{r}=0.1 c_{\text {crit }}$ (or $Q=0.1$, respectively). According to $\S 2$, the latter means that initially the disk is unstable with respect to both radial and spiral gravity perturbations. The initial distribution of particles $\left(x_{i}, y_{i}, z_{i}\right)$ was generated by means of random-number generator placing particles uniformly in the ring with rings' width $\Delta R \ll R$ (where $R$ is the 
initial radius of the system). Thus, equilibrium is governed mainly by the balance between the centrifugal and gravitational forces. It is this metaequilibrium that is to be examined for stability by simulations.

Below we describe the results of simulations of a computer model containing a sufficiently large number of particles $N=75,000$. We performed a few runs for systems containing $N=100,000$ model particles and for smaller systems containing $N=25,000$ ones. It was found that the results obtained for those systems are qualitatively indistinguishable: we did not detect in our experiments any dependence of the type $\aleph \propto N^{-1 / 2}$, where $\aleph$ is the amplitude of the density variations. The last is clearly inconsistent with Toomre's (1990) hypothesis that the spirals observed in simulations can be explained by the swing-amplified particle noise ("kaleidoscope of chaotic arm features" which are responses to the random density irregularities orbiting within the particulate disk). We advocate a way to describe the rapidly evolving structures, such as those reported in our simulations, in terms of true instabilities of Jeans perturbations (see $\S 2$ above). Also we did not find any difference between the results of simulations with or without applying the so-called quiet starts procedure to select the initial coordinates of particles. Such techniques have proven useful in obtaining realistic noise levels without the use of a large number of particles (e.g., Griv et al. 1999). Moreover, tests indicated that the results were insensitive to changes in other parameters. A test gives a good check on the numerical stability of the code as well as the accuracy of the program; the code conserves energy to within $2 \%$ during the first 5 rotations of the collisionless system.

Figure 1 shows an example of the development of spiral structures in Saturn's rings from a run. The structures grow rapidly on a dynamical time scale $\sim \Omega^{-1}$. At a time $t=4$ a filamentary spiral structure forms with a few short, tightly wound trailing arms. A typical distance between the spiral filaments is $\sim \lambda_{\text {crit }}$, indicating that perturbations with the critical wavelength have the fastest growth rate. Such a wavelength is in agreement with the theory.

In Figure 2 the time evolution of the Fourier spectrum of the pattern shown in Figure 1 is plotted. Only the azimuthal $m=1-4$ components are shown. The complex Fourier coefficients, $A$, are determined from the summation

$$
A(p, m)=\frac{1}{N} \sum_{j=1}^{N} \exp \left\{i\left[m \varphi_{j}+p \ln \left(r_{j}\right)\right]\right\},
$$

where $\left(\varphi_{j}, r_{j}\right)$ are the polar coordinates of the $j$-th particle. The pitch angle of an $m$-armed logarithmic spiral $\psi$ is given by $\psi=\arctan (p / m)$, and positive $p$ corresponds to trailing spirals and negative $p$ to leading ones. Amplitudes $|A(p, m)|$ are greatly in excess of an expectation from the level of particle noise $\sqrt{\pi / 4 N}$; true instabilities develop in the model. The result suggests that a flattened disk can be unstable to spiral gravity perturbations. The calculated spectrum is dominated by the $m=1$ harmonic. This one-armed mode may explain the global asymmetry observed in the Saturnian ring system. This logarithmic Fourier analysis shows that the peaks of the signals move to positive $p$ with increasing time, and reach maxima at $t=4$. An interesting feature is that all these modes have roughly the same pitch angle. The amplitudes of the signals grow aperiodically, supporting the assumption that we have deal with the Jeans instability. 


\section{Conclusions}

It is found that in the limiting case of rare collisions between particles the Saturnian ring disk may be unstable with respect to the Jeans perturbations. This instability of small-amplitude gravity disturbances (e.g., those produced by a spontaneous perturbation and/or a companion system) does not depend on the behavior of the particle distribution function in the neighborhood of a particular speed, but the determining factors of the instabilities are the macroscopic parameters of a self-gravitating system - the mean surface mass density, the angular velocity of regular rotation, and the dispersion of random velocities of particles. The growth rate of the almost aperiodically developing Jeans instability is large, $\Im \omega_{*} \sim \Omega$. Thus, such perturbations develop rapidly on the time scale of only a few revolutions of the system under study, leading to tightly wound spiral patterns. The typical wavelength of the most unstable perturbations is $\lambda_{\text {crit }} \approx 4 \pi \rho \approx 2 \pi h$. In Saturn's rings, $\lambda_{\text {crit }} \lesssim 100 \mathrm{~m}$. We argued that in general computer simulations confirm the predictions of the stability theory.

Acknowledgments. We thank Sverre Aarseth and David Eichler for valuable discussions. This work was supported by the Israel Science Foundation, the Israeli Ministry of Immigrant Absorption, and the Academia Sinica.

\section{References}

Bertin, G. 1980, Phys. Rep., 61, 1

Colombo, G., Goldreich, P., \& Harris, A. 1976, Nature, 264, 344

Daisaka, H., \& Ida, S. 1999, Earth Planets Space, 51, 1195

Esposito, L. W. 1993, Annu. Rev. Earth Planet. Sci., 21, 487

Franklin, F. A., \& Colombo, G. 1978, Icarus, 33, 279

Goldreich, P., \& Tremaine, S. 1982, ARA\&A, 20, 249

Griv, E. 1998, Pl\&SS, 46, 615

Griv, E., \& Chiueh, T. 1997, A\&A, 311, 1033

Griv, E., \& Chiueh, T. 1998, ApJ, 503, 186

Griv, E., Gedalin, M., Eichler, D., \& Yuan, C. 2000, Pl\&SS, 48, 679

Griv, E., Rosenstein, B., Gedalin, M., \& Eichler, D. 1999, A\&A, 347, 821

Lin, C. C., \& Lau, Y. Y. 1979, Stud. Appl. Math., 60, 97

Lin, C. C., \& Shu, F. H. 1966, Proc. Natl Acad. Sci., 55, 229

Lin, C. C., Yuan, C., \& Shu, F. H. 1969, ApJ, 155, 721

Osterbart, R., \& Willerding, E. 1995, PI\&SS, 43, 289

Richardson, D. C. 1994, MNRAS, 269, 493

Salo, H. 1992, Nature, 359, 619

Shu, F. H. 1970, ApJ, 160, 99

Shu, F. H., \& Stewart, G. R. 1985, Icarus, 62, 360.

Toomre, A. 1964, ApJ, 139, 1217

Toomre, A. 1990, in Dynamics and Interactions of Galaxies, ed. R. Wielen (Springer: Berlin), 292 